\title{
The "Molecular Grabber" method: Development of another crystalline sponge New idea for structure analysis of compounds using protein
}

\author{
T. Matsumoto ${ }^{1}$, A. Yamano ${ }^{1}$, R. Nakashima², K. Nishino ${ }^{2}$ \\ ${ }^{I}$ Rigaku Corporation, 3-9-12 Matsubara-cho, Akishima, Tokyo 196-8666, Japan, \\ ${ }_{2}^{2}$ Institute of Scientific and Industrial Research, Osaka University, 8-1 Mihogaoka, Ibaraki, Osaka 567-0047, Japan. \\ t-matumo@rigaku.co.jp
}

Molecular structure determination is beneficial for the development of medicines, aroma chemicals, and agrochemicals. Single crystal $\mathrm{X}$-ray diffraction (SC-XRD) analysis is the most powerful technique for molecular structure determination. However, SC-XRD analysis requires good quality crystals.

In fact, the biggest hurdle for SC-XRD analysis is crystallization. Crystallization trials require a large amount of highly purified target compounds. Moreover, good quality crystals for SC-XRD analysis might not be obtained despite performing tedious and timeconsuming trials. In this case, we have to abandon the direct structure determination by SC-XRD. As one way to address this situation, Fujita et al. have reported the crystalline sponge method (CS method) for the structure determination of small molecules [1]. However, as with other analysis techniques, the CS method has some limitations.

The CS method utilizes the MOF as the pre-crystallized 'container' for the analytes. The 'container' equips flexible features to fit various analytes and must have enough space to accommodate the wide variety of the molecules. In the latter mean, MOF is a large structure object with three-dimensional networks; thus, the spaces to accommodate are to have limitations in principle.

To overcome the above difficulty, we came to an idea of 'molecular grabber', utilizing protein that has a multisite binding pocket to bind a variety of types of molecules, and having characteristics on easy to crystallize, and resulted crystal gives high-resolution spots.

In this presentation, we will indicate the initial results of the "Molecular Grabber" method, utilizing RamR as the molecular grabber (Fig. 1).

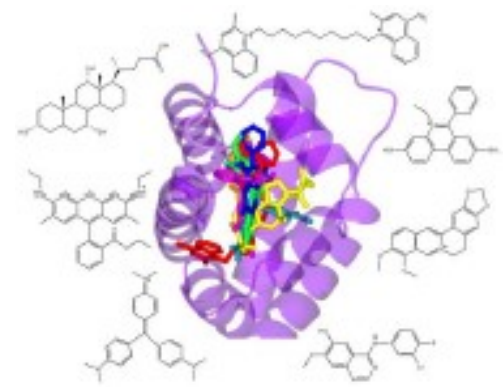

Figure 1. Superimposition of the compounds bound to RamR.

[1] Inokuma, Y., Yoshioka, S., Ariyoshi, J., Arita, T., Hirota, Y., Takada, K., Matsunaga, S., Rissanen, K. \& Fujita, M. (2013). Nature $495,461-467$.

[2] Matsumoto, T., Nakashima, R., Yamano, A. \& Nishino, K. (2019). Biochem. Biophys. Res. Commun. 518, $402-408$.

Keywords: Molecular Grabber; crystalline sponge method; multisite binding pocket 